\title{
MiRNAs are Unlikely to be Involved in Retinoid Receptor Gene Regulation in Pancreatic Cancer Cells
}

\author{
Shuai Yin ${ }^{\mathrm{a}}$ Tim Bleul $^{\mathrm{a}}$ Yifan Zhu ${ }^{\mathrm{b}, \mathrm{c}}$ Orkhan Isayeveved Jens Werner ${ }^{\mathrm{a}, \mathrm{e}}$ \\ Alexandr V. Bazhina,e \\ aDepartment of General, Visceral, and Transplantation Surgery, University Hospital of the LMU, \\ Munich, Germany; bepartment of Oncology, Henan University Huaihe Hospital, Kai Feng, P.R. China; \\ International Joint Research Laboratory for Cell Medical Engineering of Henan, P.R.China; ${ }^{\mathrm{d} D e p a r t m e n t}$ \\ of Histology, Embryology and Cytology, Azerbaijan Medical University, Baku, Azerbaijan; ${ }^{\mathrm{e}}$ German \\ Cancer Consortium (DKTK), Partner Site Munich, Germany
}

\section{Key Words}

Retinoid receptors $\cdot$ MiRNA - Pancreatic cancer

\begin{abstract}
Background/Aims: Retinoid receptors and retinoic acid were reported to be down-regulated in pancreatic duct adenocarcinoma (PDAC) compared to normal pancreas. Yet the mechanism of the down-regulation of retinoid receptors is not well defined. The aim of this study was to find out whether selected dysregulated miRNAs in PDAC are responsible for the decreased level of retinoid receptors. Methods: Bioinformatics, real-time PCR, western blot analysis as well as molecular manipulation with miRNA in cells of PDAC were carried out. Results: We first performed bioinformatics research to identify conserved target sequences for deregulated miRNAs within the 3'UTR region of retinoid receptor mRNA. This research revealed binding sites for miR-138, $-27 a,-27 b,-206,-613,-9-5 p,-27 a / b-3 p$ and $-27 a$. Next, we investigated the expression of selected retinoid receptors and miRNAs in PDAC cell lines and in the Human Pancreatic Duct Epithelial (HPDE) cell line. Further, we investigated the effects of modifying expression levels of selected miRNAs using miRNA inhibitors or mimics. We demonstrated that none of these miRNAs can target the selected retinoid receptors in vitro. Conclusions: miR-27a, miR-27b, miR-9, miR10a and miR-10b were up-regulated in PDAC cells compared to HPDE cells. The up-regulation of these miRNAs was not responsible for the down-regulation of RAR $\alpha, R A R \beta, R X R \alpha$ and RXR $\beta$ in PDAC cells.

(C) 2017 The Author(s)

Published by S. Karger AG, Basel

\section{Introduction}

Gene expression in benign and malignant cells can be regulated on various levels, such as epigenetic regulation (e.g. histone modifications, DNA methylation), transcriptional


control (e.g. promoter regulation by transcription factors) or translational control by non-coding RNAs. miRNAs are small non-coding endogenous RNAs that regulate multiple biological processes by mechanisms that are still not fully understood [1]. These regulatory elements are first transcribed and then processed by Dicer and Drosha complexes into 21-23nt mature miRNAs [2]. The miRNA is incorporated into the RNA-induced silencing complex (RISC) containing Dicer and many other associated proteins including members of the Argonaute (Ago) protein family [3]. RISC regulates posttranscriptional mRNA expression (i.e. translation suppression) typically by binding to the 3' untranslated region (3'-UTR) of the complementary mRNA sequence, preventing the recognition of cap by eIF4E and subsequent binding of translational factors [4]. Therefore, there is some evidence proving that the translation activation by miRNAs is regulated through direct or indirect ways [5]. 3'UTR is not a unique target sequence for miRNAs. It has been shown that miRNAs also efficiently bind to the 5'UTR [6, 7] leading to activation [8-10] or repression [11,12] of translation. However the precise mechanism by which miRNA binds to the 5'UTR is still to be deciphered.

Retinoic acid, the metabolite of vitamin A, is important for growth and differentiation of both healthy and cancerous cells. All-trans retinoic acid (ATRA), the major physiologically active form of vitamin A, regulates the expression of many genes [13]. Physiological effects of retinoid are mainly mediated by two families of nuclear receptors: the retinoic acid receptor (RAR) and the retinoid X receptor (RXR). RAR and RXR are both ligand-dependent transcription factors, which belong to the nuclear hormone receptor superfamily. The RAR and RXR family comprise several isotypes: $\operatorname{RAR} \alpha, \operatorname{RAR} \beta, \operatorname{RAR} \gamma$ and $\operatorname{RXR} \alpha \operatorname{RXR} \beta, \operatorname{RXR} \gamma$ [13].

Pancreatic ductal adenocarcinoma (PDAC) is one of the deadliest cancers in the world. At present, only multimodal treatment including surgical resection can prolong survival of patients with this disease [14]. The reasons for this poor prognosis are early dissemination, lack of early specific symptoms and late diagnosis [15]. Additionally, PDAC is highly resistant to chemo- and targeted therapy, and withstands immunotherapy [16]. Therefore, new therapeutic approaches against PDAC are urgently needed. It has been shown that different miRNA promote growth and proliferation of PDAC cells [17-20] and predict poor clinical outcome of patients with PDAC [21].

Recently, ATRA and its derivatives have been reported to inhibit proliferation and migration of various cancer types including PDAC [22, 23]. Our previous studies showed that the concentration of ATRA and its derivatives as well as retinoid receptor expression are reduced in PDAC cancer tissues compared to normal pancreatic tissue [24, 25]. More importantly, the reduced expression of retinoid receptors correlates with the expression of markers of epithelial to mesenchymal transition and stemness of PDAC cells [24]. Besides, the expression of RARA and RXRB is found to be associated with a better overall survival in PDAC patients. Accordingly, the reduced level of retinoids and their receptors is an important feature of PDAC [24]. However, the mechanism of the down-regulation of retinoid receptors is not well defined.

The present study aimed to identify novel oncogenic-suppressive miRNAs as the regulators of retinoid receptors in PDAC.

\section{Materials and Methods}

\section{Materials}

Ampicillin, chloroform, isopropyl alcohol, ethanol, 0.4\% trypan blue solution, EDTA, TEMED, ammonium persulfate and DMSO were purchased from Sigma (Darmstadt, Germany). Agarose was obtained from Gibco BRL (Idstein, Germany). Odyssey® Blocking Buffer was purchased from LI-COR (Lincoln, NE, USA). Kits: HiperFect transfection, RNeasy Mini, miScript II RT, miScript SYBR Green PCR, QuantiTect Reverse Transcription, QuantiTect SYBR Green PCR QuantiTect Primer Assays, miScript Primer Assays and QIAzol Lysis Reagent were from Qiagen (Hilden, Germany). Anti-RAR and anti-RXR antibodies were obtained from SantaCruz (Dallas, TX, USA). Anti-MET antibody was from Sigma (Darmstadt, Germany). IRDye® 680LT 
Yin et al.: Mirna and Retinoid Receptors in PDAC

Goat anti-Mouse, IRDye $₫$ 800CW Donkey anti-Rabbit, IRDye $\AA$ 680CW Goat anti-Rabbit and IRDye® 800CW Donkey antiMouse antibodies were purchased from LI-COR (Lincoln, NE, USA). All miRNA products were purchased from Qiagen (Hilden, Germany). miRNA products and primers used are listed in the Table 1.

\section{Cell lines}

Human PDAC cell lines: MiaPaCa-2 and Panc-1 derived from primary mesenchymal tumors and BxPc-3 and DanG derived from primary epithelial tumors were chosen for experiments. All cell lines were obtained from ATCC (Manassas, VA, USA). PDAC cells were maintained as a monolayer. Prior
Table 1. miRNAs products and primers

\begin{tabular}{ll}
\hline miRNA mimic or inhibitors & \multicolumn{1}{c}{ Sequence (5'-3') } \\
\hline Syn-hsa-miR-9-5p miScript miRNA Mimic & 5'UCUUUGGUUAUCUAGCUGUAUGA \\
Syn-hsa-miR-27a-3p miScript miRNA Mimic & 5'UUCACAGUGGCUAAGUUCCGC \\
Syn-hsa-miR-27a-5p miScript miRNA Mimic & 5'AGGGCUUAGCUGCUUGUGAGCA \\
Syn-hsa-miR-27b-3p miScript miRNA Mimic & 5'UUCACAGUGGCUAAGUUCUGC \\
Syn-hsa-miR-27b-5p miScript miRNA Mimic & 5'AGAGCUUAGCUGAUUGGUGAAC \\
Anti-hsa-miR-9-5p miScript miRNA Inhibitor & 5'UCUUUGGUUAUCUAGCUGUAUGA \\
Anti-hsa-miR-27a-3p miScript miRNA Inhibitor & 5'UUCACAGUGGCUAAGUUCCGC \\
Anti-hsa-miR-27a-5p miScript miRNA Inhibitor & 5'AGGGCUUAGCUGCUUGUGAGCA \\
Anti-hsa-miR-27b-3p miScript miRNA Inhibitor & 5'UUCACAGUGGCUAAGUUCUGC \\
Anti-hsa-miR-27b-5p miScript miRNA Inhibitor & 5'AGAGCUUAGCUGAUUGGUGAAC \\
miRNA negative control Oligos & \\
Hs_miR-9_1 miScript Primer Assay & \\
Hs_miR-27a_1 miScript Primer Assay & \\
Hs_miR-27b_2 miScript Primer Assay & \\
Hs_miR-138_1 miScript Primer Assay & \\
Hs_miR-206_1 miScript Primer Assay & \\
Hs_miR-613_1 miScript Primer Assay & \\
Hs_RARA_1_SG QuantiTect Primer Assay (QT00095865) & \\
Hs_RARB_1_SG QuantiTect Primer Assay (QT00062741) & \\
Hs_RARG_1_SG QuantiTect Primer Assay (QT00000987) & \\
Hs_RXRA_1_SG QuantiTect Primer Assay (QT00005726) & \\
Hs_RXRB_1_SG QuantiTect Primer Assay (QT00061117) & \\
Hs_RXRG_1_SG QuantiTect Primer Assay (QT00007238) & \\
\hline
\end{tabular}

to passaging, trypsin and media were warmed up in a $37^{\circ} \mathrm{C}$ water bath. Cells were cultured in Dulbecco's modified Eagle's medium (DMEM) supplemented with 10\% fetal bovine serum and 1\% penicillin/streptomycin and incubated at $37^{\circ} \mathrm{C}$ in a humidified atmosphere of $5 \% \mathrm{CO}_{2} / 95 \%$ air. Cells were passaged once or twice a week when a confluence of $80 \%$ was reached. Normal HPDEcells (kindly provided by Dr. Kong Bo, Technical University Munich, Germany) were maintained in Keratinocyte Basal Medium with supplements (Lonza, Clonetics KBM, Basel, Switzerland). The flask was incubated at $37^{\circ} \mathrm{C}$ in a humidified atmosphere of $5 \% \mathrm{CO} 2 / 95 \%$ air. Culture medium was replaced every two to three days. Cells were passaged when a confluence of $80 \%$ is reached. All cells were routinely checked for mycoplasma in conditioned cell medium using PCR according to internal SOPs and authenticated commercially by IDEX BioResearch (Ludwigsburg, Germany).

\section{Total RNA and miRNA isolation from cultivated cells}

Total RNA from cells was isolated using RNeasy Mini Kit following the manufacturer's instruction and described elsewhere [26]. RNA was quantified using a GeneQuant pro spectrophotometer (Eppendorf, Hamburg, Germany). The quality of isolated mRNA was evaluated by the A260/A280 value. To assess of RNA quality, the samples of total RNA were diluted 1:50, applied on the RNA 6000 Nano LabChip (Agilent Technologies, Palo Alto, CA) and analyzed with the Agilent Bioanalyzer 2100 (Aglient Technologies, Palo Alto, CA). All samples had RNA Integrity Numbers between 9.5 and 10 .

Total miRNA from cells was isolated using QIAzol (Qiagen, Hilden, Germany) following the manufacturer's instruction (miRNeasy Mini kit, Qiagen, Hilden, Germany). In brief, cells were trypsinized at $37^{\circ} \mathrm{C}$, collected and centrifuged at $300 \times \mathrm{g}$ for 5 minutes. The cell pellet was then washed with cold $1 \times$ PBS 3 times. Cells were then pelleted in a $1.5 \mathrm{ml}$ Eppendorf tube. By adding $700 \mu \mathrm{l}$ QIAzol lysis reagent, cells were lysed after incubation at room temperature for 5 minutes. Then $140 \mu$ chloroform was added and the tube was shaken vigorously for 15 seconds. After incubation at room temperature for 3 minutes, the tube was centrifuged at $8000 \times \mathrm{g}$ at $4^{\circ} \mathrm{C}$ for $15 \mathrm{~min}$. The upper aqueous phase which contains miRNA was transferred into a new collection tube. Then 1.5 volumes of $100 \%$ ethanol was added and well mixed, before loading onto a RNeasy Mini spin column in a $2 \mathrm{ml}$ collection tube for centrifugation at $8000 \times \mathrm{g}$ (RT, $1 \mathrm{~min}$ ). The RNeasy Mini spin column which contains miRNAs was then washed with $700 \mu$ l RWT Buffer (included in the Qiagen kit) once and $500 \mu \mathrm{l}$ RPE Buffer (included in the Qiagen kit) twice. 30-50 $\mu$ l RNase-free water was then added directly onto the RNeasy Mini spin column membrane and miRNAs were obtained by centrifugation at 8000 $\times \mathrm{g}$ for $2 \mathrm{~min}$. RNA was quantified using a spectrophotometer. The quality of isolated miRNA was evaluated by the A260/A280 value. To determine the content of miRNA, each sample was diluted 1:50, applied on the Small RNA Nano LabChip (Agilent Technologies, Palo Alto, CA) and analyzed with the Agilent Bioanalyzer 2100 (Agilent Technologies, Palo Alto, CA). The ratio miRNA to small RNA was in the range 1-7\%. 


\section{Cellular Physiology Cell Physiol Biochem 2017;44:644-656 \begin{tabular}{l|l|l} 
and Biochemistry 10.1159/000485276 & $\begin{array}{l}\text { (c) } 2017 \text { The Author(s). Published by S. Karger AG, Basel } \\
\text { www.karger.com/cpb }\end{array}$
\end{tabular}}

Yin et al.: Mirna and Retinoid Receptors in PDAC

\section{Real time PCR analysis of mature miRNAs}

Generation of the first strand cDNA from miRNA was done using miScript II RT Kit (Qiagen, Hilden, Germany). For this purpose, an equal amount of miRNA (500 ng) from each miRNA sample (in a final volume of $20 \mu \mathrm{l}$ containing universal primer) was added into each reaction at the last step. The miRNA was placed on ice and the reverse transcription reaction was set up by mixing the following components from the kit: $4 \mu \mathrm{L}$ of $5 \mathrm{x}$ miScript HiSpec Buffer, $2 \mu \mathrm{L}$ of 10x Nucleics Mix, $2 \mu \mathrm{L}$ of miScript Reverse Transcriptase Mix, variable volume of RNA template as well as RNase-free water. The end volume of each sample was $20 \mu \mathrm{L}$. The reagents were gently mixed and briefly centrifuged. Reverse transcription was carried out by incubation at $37^{\circ} \mathrm{C}$ for 60 minutes with the reaction terminated by incubation at $95^{\circ} \mathrm{C}$ for 5 minutes. After the reaction, each tube was diluted with $200 \mu \mathrm{L}$ RNase-free water for later use in RT-PCR.

Expression of mature miRNAs was determined by miScript miRNA PCR Array (Qiagen, Hilden, Germany). For this purpose, $1 \mu \mathrm{l}$ cDNA was used per $25 \mu \mathrm{l}$ reaction mixture containing $12.5 \mu \mathrm{l}$ of $2 \mathrm{x}$ QuantiTect SYBR Green PCR Master Mix, $2.5 \mu \mathrm{l}$ of 10x miScript Universal Primer, $2.5 \mu \mathrm{l}$ of 10x miScript Primer Assay and variable volume of RNase-free Water in a 96-well plate. The 96-well plate was briefly centrifuged for 1 minute to remove any bubbles. The reaction was then carried out for 40 cycles in StepOne ${ }^{T M}$ Real-Time PCR System (Thermo Fisher, Darmstadt, Germany). The program included the following steps: 15 min initial activation at $95^{\circ} \mathrm{C}, 15 \mathrm{~s}$ denaturation at $94^{\circ} \mathrm{C}, 30 \mathrm{~s}$ annealing at $55^{\circ} \mathrm{C}$ and finally $30 \mathrm{~s}$ extension at $70^{\circ} \mathrm{C}$. U6snRNA was used as a house-keeping control for normalization. $2^{-\Delta \Delta c t}$ method was used to calculate the fold change of miRNA expression using the equation $2^{-\Delta \Delta C t}=2^{-[C t \text { gene of interest }-C t \text { internal control) }) \text { sample } A-(C t \text { gene of interest }-C t \text { internal }}$ control)sample B] as described elsewhere [27], where internal control refers to the house-keeping control, sample A was a PDAC cell line and sample B was the HPDE cell line.

\section{SYBR Green quantitative PCR for retinoid receptor}

Genomic DNA was eliminated from the RNA samples before initiating reverse transcription using the QuantiTect Reverse Transcription Kit (Qiagen, Hilden, Germany) with 500 ng of RNA from each sample. The reaction mixes for genomic DNA elimination (total volume of $14 \mu \mathrm{l}$ ) included $2 \mu \mathrm{l}$ of 7x gDNA Wipeout Buffer and variable volume of Template RNA and RNase-free water. The reaction tubes were put directly on ice after incubation for $2 \mathrm{~min}$ at $42^{\circ} \mathrm{C}$. After that, $6 \mu \mathrm{l}$ of reverse-transcription master mix ( $1 \mu \mathrm{l}$ of Quantiscript Reverse Transcriptase, $4 \mu \mathrm{l}$ of $5 \mathrm{x}$ Quantiscript RT Buffer and $1 \mu$ l of RT primer mix) was prepared per sample on ice. To each reverse transcription master mix tube, $14 \mu \mathrm{l}$ of Template RNA after genomic DNA elimination reaction was added. In a PCR machine, reverse transcription was carried out for $15 \mathrm{~min}$ at $42^{\circ} \mathrm{C}$ and then Quantiscript Reverse Transcriptase was inactivated after $3 \mathrm{~min}$ at $95^{\circ} \mathrm{C}$.

Expression of the retinoid receptor was determined by QuantiFast SYBR Green PCR kit (Qiagen, Hilden, Germany) following the manufacturer's instruction and described elsewhere [24]. Briefly, $1 \mu \mathrm{l}$ of cDNA synthesized before was added to reaction mix in 96-well plate. The reaction mixture consisted of the following components: $12.5 \mu \mathrm{l}$ 2x QuantiFast SYBR Green PCR Master Mix, $2.5 \mu \mathrm{l} 10 \mathrm{x}$ QuantiTect Primers and $9 \mu \mathrm{l}$ RNAse-free water. The 96-well plate was briefly centrifuged for 1 minute to remove any bubbles. The cycling program was carried out in a StepOne $\mathrm{P}^{\mathrm{TM}}$ Real-Time PCR System (Thermo Fisher, Darmstadt, Germany) with the following parameters: 5 min PCR initial activation step at $95^{\circ} \mathrm{C}$ followed by 40 twostep cycles of $10 \mathrm{~s}$ denaturation at $95^{\circ} \mathrm{C}$ and $30 \mathrm{~s}$ combined annealing/ extension at $60^{\circ} \mathrm{C}$. GAPDH mRNA was used as a house-keeping gene for normalization. $2^{-\Delta c t}$ method was used to calculate the fold change of retinoid gene expression as described above.

\section{Transfection of miRNAs}

We used HiPerFect Transfection Reagent (Qiagen, Hilden, Germany) to transfect miRNAs into pancreatic cancer cells. Transfection was done following manufacturer's manual. The initial step was to determine optimal miRNA transfection conditions for different cells. Briefly, $1 \times 10^{5}$ cells were suspended in $0.5 \mathrm{ml}$ of growth medium and seeded into one well of a 24-well plate. Then appropriate amounts of miRNA and HiperFect Transfection Reagent were added into $100 \mu \mathrm{l}$ culture medium without serum. The mixture was vortexed and incubated for 5 to $10 \mathrm{~min}$ at room temperature to allow the formation of transfection complexes. Transfection was achieved by adding the mixture dropwise onto the cells in a well. The plate was gently swirled and incubated under normal growth condition $\left(5 \% \mathrm{CO}_{2}, 37^{\circ} \mathrm{C}, 90 \%\right.$ humidity) for 48 to $72 \mathrm{~h}$. After the incubation for 48 to 72 hours, cells were collected and miRNAs were isolated with the protocol as described before. The level of target miRNA in transfected cells was determined by RT-PCR and 


\section{Cellular Physiology Cell Physiol Biochem 2017;44:644-656 \begin{tabular}{l|l|l} 
and BOI: 10.1159/000485276 & $\begin{array}{l}\text { (c) 2017 The Author(s). Published by S. Karger AG, Basel } \\
\text { www.karger.com/cpb }\end{array}$
\end{tabular} \\ Yin et al.: Mirna and Retinoid Receptors in PDAC}

compared with non-transfected cells. The same protocol was used for transfection of miRNA mimics and miRNA inhibitors.

\section{Western blot analysis}

Western blotting was performed as described elsewhere [26]. Briefly, cells grown in a 24 well-plate were washed with cold phosphate-buffered saline (PBS buffer) three times and lysed in RIPA extraction buffer (150 mM sodium chloride, 1\% NP-40 or Triton X-100, 0.5\% sodium deoxycholate, 0.1\% SDS (sodium dodecyl sulfate) and 50mM Tris at pH 8.0) on ice for $15 \mathrm{~min}$. The lysates were centrifuged at 14, $000 \mathrm{rpm}$ for $20 \mathrm{~min}$ and the supernatant containing the total cell extract was stored at $-80^{\circ} \mathrm{C}$ until used. The extracted protein was quantified using the BCA Protein assay kit (Thermo Fisher, Darmstadt, Germany). The proteins resolved by SDS-PAGE were transferred onto a PVDF membrane by semi-dry electrophoretic transfer. The membrane was first wetted in $100 \%$ methanol for 5 seconds and then equilibrated in transfer buffer. The blot was assembled on a semi-dry blotting apparatus from anode to cathode as follow: 2 Whatman papers soaked in transfer buffer, PVDF membrane, gel and finally 2 Whatman papers. Transfer of proteins was done for 1 hour by applying $1.8 \mathrm{~V}$ per $\mathrm{cm}$ square of the blot. The membrane was then blocked for 1 hour at room temperature in TBS containing $0.1 \%$ Tween 20 and 5\% BSA. The membrane was then incubated overnight with the primary antibody in blocking solution. Following three 10-min washes with $0.1 \%$ Tween 20 in TBS (TBST) the blot was incubated for 1 hour at room temperature with the fluorescence-conjugated secondary antibody diluted in Odyssey® Blocking Buffer (LI-COR, Lincoln, NE, USA) for $1 \mathrm{~h}$ at room temperature in the dark. The membrane was then washed 3 times at 10 min intervals with TBST buffer. Finally, the protein band was visualized in the Odyssey Reader machine (LI-COR, Lincoln, NE, USA). Densitometric values of the bands were calculated and normalized with respect to background by using GelAnalyzer 2010 software.

\section{Bioinformatics analysis of the miRNA target}

To identify potential target sequences in the 3'UTR of retinoid receptor mRNA, various public database websites were utilized: miRNA.org (http://www.microrna.org/microrna/getGeneForm.do), miRDB (http:// www.mirdb.org/miRDB/__ and TargetScanHuman (http://www.targetscan.org/vert_71/). For miRDB, miRNA target prediction program is based on support vector machines (SVMs), CLIP-ligation studies, a large microarray training dataset and a set of functional miRNAs annotated by integrating computational analyses with literature mining [28-30]. For Targetscan, 14 features (e.g., site type, $3^{\prime}$-supplementary pairing, local AU content, and distance from the closest $3^{\prime}$-UTR end), two miRNA features (TA and SPS) were selected and combined to develop the context++ model of miRNA targeting efficacy [31]. PicTar is also a computational method base on nuclMap, optimal free energy, alignments of highly probable nuclei and established score system [32]. Specific complementary target sequences were recorded for all predicted targets. In addition, another public database (miRTarBase, http://mirtarbase.mbc.nctu.edu.tw/) of experimentally validated miRNA targets was utilized to combine with the information of already published data.

\section{Statistical analysis}

Statistical analyses were performed using either Statistical Package for the Social Sciences (IBM SPSS Statistic 24) software or GraphPad Prism 7 (GraphPad Software, Inc.) software. Values were presented as means \pm SEM. Different statistical tests, such as one-way ANOVA and Pearson's correlation were utilized depending on specific data types, as indicated in the figures. Values were considered significantly different when p-values were less than 0.05 .

\section{Results}

\section{Prediction of retinoid receptor sequences targeted by miRNAs}

In our previous study, we showed that the down-regulation of retinoid receptors in PDAC is negatively correlated with patient survival, which indicates that retinoid receptors could function as tumor suppressors [24]. To investigate whether retinoid receptor mRNAs are targeted by specific miRNAs, the first step is to predict the specific binding sites in the 3'UTRs. For this purpose, we integrated the predictions from different databases (Targetscan, mirbase, Pictar and miRDB) to increase the probability of finding all potential miRNA targets. 


\section{Cellular Physiology Cell Physiol Biochem 2017;44:644-656 \begin{tabular}{ll|l} 
DOI: 10.1159/000485276 & $\begin{array}{l}\text { @ 2017 The Author(s). Published by S. Karger AG, Basel } \\
\text { www.karger.com/cpb }\end{array}$ \\
and Biochemistry Published online: November 23, 201
\end{tabular}}

Table 2. Prediction of miRNA targets. ${ }^{\text {aPredicted by }}$

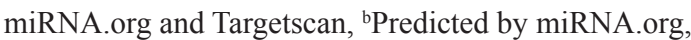

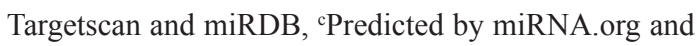
miRDB, dPredicted by miRNA.org and miRDB, ${ }^{\mathrm{e} P r e-}$

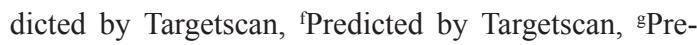
dicted by miRNA.org

As these predictive databases are based on different algorithms, the prediction of miRNA target sequences from a single database could result in high numbers of false positives. We used both theintersection or the union of miRNA targets predicted by the different algorithms. As the purpose of this research was to find the dysregulated miRNAs that can target retinoid receptors in PDAC, we first recruited dysregulated miRNAs and then used different algorithms to predict potential candidate miRNAs that target retinoid receptors. If the selected miRNA was predicted to target any of these receptors by any of these algorithms, it was recruited for validation in the next step. To avoid missing any possible miRNA targets, we integrated the predictions from the different algorithms. Some of the miRNAs, like miR-27a and miR-27b, were predicted to target more than one retinoid receptor by more than one algorithm (Table 2). In the aggregate, miR-138, miR-206, miR613, miR-9, miR-27a/b and miR-613 can potentially target retinoid receptors.

To identify the specific targeted sites, each mRNA of the retinoid receptor was screened for complementarity to seed

\begin{tabular}{ccccccc}
\hline & miR-9a & miR-10 & miR-27a/b & miR-138 & miR-206 & miR-613 \\
\hline $\operatorname{RAR} \boldsymbol{\alpha}$ & No & No & Yes $^{\mathrm{a}}$ & Yes $^{\mathrm{b}}$ & No & No \\
$\operatorname{RAR} \boldsymbol{\beta}$ & No & No & No & No & Yes $^{\mathrm{c}}$ & Yes $^{\mathrm{d}}$ \\
$\operatorname{RXR} \boldsymbol{\alpha}$ & Yes $^{\mathrm{e}}$ & No & Yes & No & No & No \\
$\operatorname{RXR} \boldsymbol{\beta}$ & No & No & Yesg & No & No & No \\
\hline
\end{tabular}

\begin{tabular}{|c|c|}
\hline $\begin{array}{l}\text { 3' GCCGGACUAAGUGUUGUGGUCGA } \\
\quad \text { I I I I I IIIIIIIII } \\
314: 5 \text { UCACCACAUCUUCAUCACCAGCA }\end{array}$ & $\begin{array}{l}\text { 5' has-miR-138 } \\
\text { 3' RARA }\end{array}$ \\
\hline $\begin{array}{l}3^{\prime} \text { CGCCUUGAAUCGGUGACACUU } \\
\text { III I I I I III } \\
726: 5^{\circ} \text { UUUUCUCUUUAAACUGUGAa }\end{array}$ & $\begin{array}{l}\text { 5' has-miR-27a } \\
\text { 3' RARA }\end{array}$ \\
\hline 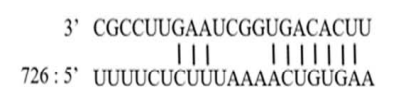 & $\begin{array}{l}\text { 5' has-miR-27b } \\
\text { 3' RARA }\end{array}$ \\
\hline 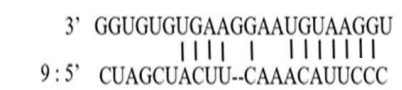 & $\begin{array}{l}\text { 5' has-miR-206 } \\
\text { 3' RARB }\end{array}$ \\
\hline  & $\begin{array}{l}\text { 5' has-miR-613 } \\
\text { 3' RARB }\end{array}$ \\
\hline $\begin{array}{r}\text { 3' UAUGUCGAUCUAUUGGUUUCU } \\
\text { I I I I I11 } \\
623: 5 \text { ' GGUAGCCCCUUUUUCCAAAGA }\end{array}$ & $\begin{array}{l}\text { 5' has-miR-9-5p } \\
\text { 3' RXRA }\end{array}$ \\
\hline $\begin{array}{l}\text { 3' CGCCUUGAAUCGGUGACACUU } \\
\text { 1 I I I I I I I } \\
\text { 3804:5' CUUCCUGUGACUGACUGUGAA }\end{array}$ & $\begin{array}{l}5^{\prime} \text { has-miR-27a/b-3p } \\
\text { 3' RXRA }\end{array}$ \\
\hline  & $\begin{array}{l}5 ' \text { has-miR-27a } \\
\text { 3' RXRB }\end{array}$ \\
\hline
\end{tabular}

Fig. 1. Target sequence of selected miRNAs within 3' UTR of retinoid receptors predicted by integrated database. sequences of selected miRNAs. As a result, several $100 \%$ matches to target sequences were found: (1) miR-138 targets nt330-337 of the RARA-3'UTR; (2) miR-27a and miR-27b target nt740-747 of the RARA-3'UTR; (3) miR206 targets nt22-30 of the RARB-3'UTR; (4) miR-613 targets nt21-29 of the RARB-3'UTR; (5) miR-9-5p targets nt637-644 of the RXRA-3'UTR; (6) miR-27a/b-3p targets nt2818-2826 of the RXRA-3'UTR (Fig. 1). In addition, a partial match to a target sequence was also identified: miR-27a targets nt177-196 (data not shown).

\section{Basal expression of selected miRNAs and retinoid receptors in PDAC cell lines compared to} HPDE cells

To find out whether there are correlations between the expression of selected miRNAs and these retinoid receptors, we investigated the basal expression levels of miR-27a and b, $-206,-613,-138,-9,-10$ a and $b$ in a HPDE cell line and 4 PDAC cell lines: BxPC-3, Panc1, DanG, MiaPaCa-2. As one can see in Fig. 1, expression levels of these selected miRNAs varied considerably. Specifically, miR-27a increased by nearly 3 and 2 fold in BxPC-3 and MiaPaCa-2 cells respectively, compared to the expression level in HPDE cells. In contrast, the expression of miR-27a slightly decreases in PanC-1 and DanG cells (Fig. 2). Interestingly, the 
Fig. 2. miRNA expression in PDAC and HPDE cell lines determined by RT-qPCR. $500 \mathrm{ng}$ of total RNA extracted from each cell line was used to determine miRNA expression levels by RT-qPCR. Relative expression levels were calculated by normalisation to the U6 expression level. An adjustment factor was applied to all miRNA relative expression levels so that the average value in HPDE cells was arbitrarily set to 1.0. The signifi-


cant fold changes of miRNA in

PDAC cell lines compared with HPDE cells are indicated with an asterisk $\left({ }^{*}\right)$ or double asterisks $(* *)$ when p values are $<0.05$ or $<0.01$ respectively after performing one-way ANOVA. n.s. indicates that there is no significant difference between the indicated PDAC group and the HPDE group.

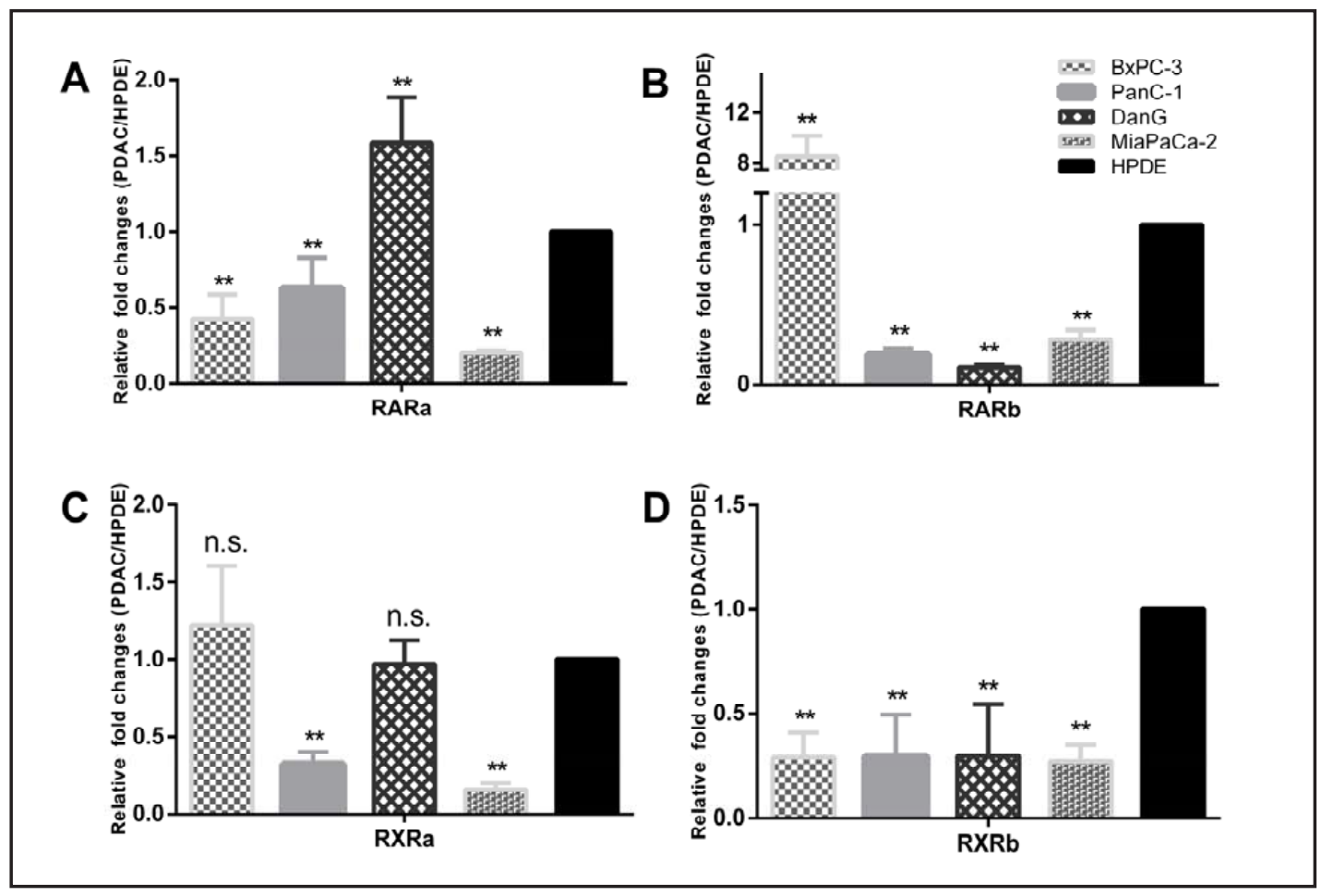

Fig. 3. Messenger RNA expression of retinoid receptors in PDAC cell lines and HPDE cell line determined by RT-qPCR. 500 ng of total RNA extracted from each cell line was used to determine mRNA expression levels by RT-qPCR. Relative expression levels were calculated by normalization to the GAPDH expression level. An adjustment factor was applied to all miRNA relative expression levels so that the average value in HPDE was arbitrarily set to 1.0. The significant fold changes of mRNAs in PDAC cell lines compared with HPDE cells are indicated with double asterisks $\left(^{* *}\right)$ when $\mathrm{p}$ values are $<0.01$ after performing one-way ANOVA. n.s. indicates that there is no significant difference between the indicated PDAC group and the HPDE group.

basal expression of miR-206, miR-613 and miR-138 are significantly decreased in PanC-1, DanG and MiaPaCa-2 cells, but not significantly changed in BxPC-3 cells compared to the level in HPDE cells. In addition, miR-9, miR-10a and miR-10b are all high expressed in PanC- 
Table 3. Correlations between expression levels of selected miRNAs and retinoid receptors in HPDE and PDAC cell lines. ${ }^{\text {a }}$ Spearman's correlation coefficient, ${ }^{\text {b }}$ Spearman's rank correlation test, *Significantly correlated with Spearman's correlation coefficient $>+0.50$ or $<-0.50$ and $P<0.05$

\begin{tabular}{lllll}
\hline & RAR $\boldsymbol{\alpha}$ & RAR $\boldsymbol{\beta}$ & $\mathbf{R X R} \boldsymbol{\alpha}$ & RXR $\boldsymbol{\beta}$ \\
\hline \multirow{2}{*}{ miRNA27a } & $-0.8 \mathbf{a}$ & $+0.900^{*}$ & +0.300 & -0.600 \\
& $0.104 \mathbf{b}$ & 0.037 & 0.624 & 0.285 \\
miRNA206 & +0.100 & +0.700 & $+0.900^{*}$ & +0.300 \\
& 0.873 & 0.188 & 0.037 & 0.624 \\
miRNA613 & +0.500 & +0.500 & $+0.900^{*}$ & +0.700 \\
& 0.391 & 0.391 & 0.037 & 0.188 \\
miRNA138 & +0.300 & +0.600 & $+1.000^{*}$ & +0.400 \\
& 0.624 & 0.285 & 0.000 & 0.505 \\
miRNA9 & +0.700 & -0.600 & 0.000 & +0.600 \\
& 0.188 & 0.285 & 1.000 & 0.285 \\
miRNA10a & +0.051 & -0.154 & +0.154 & -0.103 \\
& 0.935 & 0.805 & 0.805 & 0.870 \\
miRNA10b & -0.462 & +0.359 & +0.051 & -0.308 \\
& 0.434 & 0.553 & 0.935 & 0.614 \\
miRNA27b & +0.000 & +0.500 & +0.700 & +0.100 \\
& 1.000 & 0.391 & 0.188 & 0.873 \\
\hline
\end{tabular}

Fig. 4. Expression of retinoid receptors in Panc-1 and DanG cells transfected with miR27a, miR27b or miR9. (A) RAR $\alpha$, (B) RAR $\beta$, (C) RXR $\alpha$ and (D) RXR $\beta .25 \mu \mathrm{g}$ of total protein extracted from Panc-1 and DanG cell lines were electrophoresed in 10\% SDS-PAGE and followed by Western blotting. The membrane was incubated with rabbit polyclonal anti-RARa antibody as the primary antibody and IRDye ${ }^{\circledR}$ $680 \mathrm{CW}$ Goat anti-Rabbit as the secondary antibody. GAPDH quantity was used as the loading control. Densitometric values of the bands were calculated and normalized against background using GelAnalyzer 2010 software. Relative intensities (i.e. density ratio of target protein/ GAPDH) are represented in bar diagrams, with each bar depicting the mean \pm S.D. from 2 different analyses.

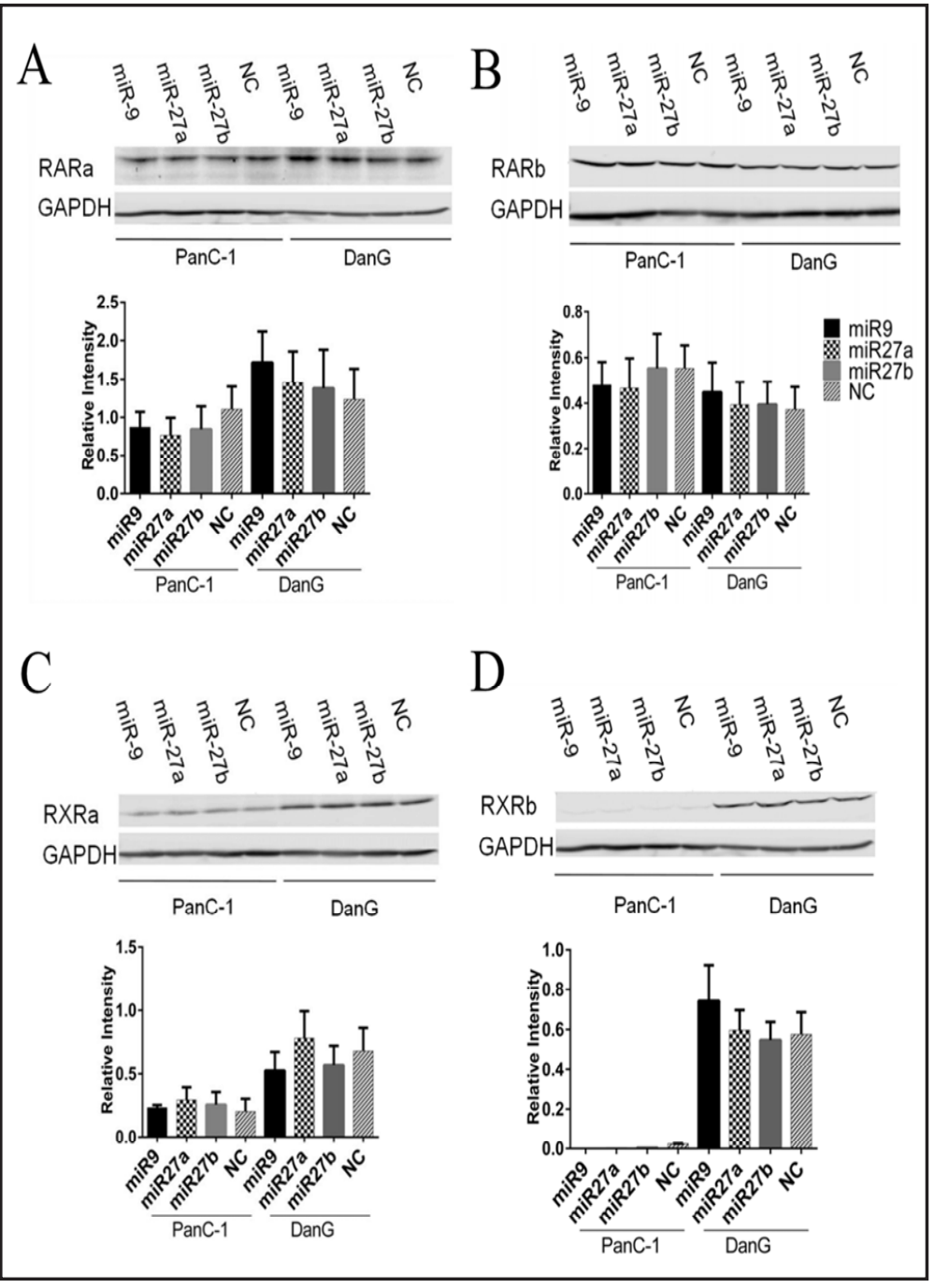

1 cells. For miR-27b, the expression levels increase significantly in BxPC-3 and PanC-1 cells with a fold change of 5 and 1.8 respectively, but decrease by $96 \%$ percent in MiaPaCa- 2 cells (Fig. 2). 
Fig. 5. Expression of MET in Panc- 1 and DanG cells transfected with miR27a. (A) $25 \mu \mathrm{g}$ total protein extracted from Panc-1 and DanG cell lines were electrophoresed in 10\% SDS-PAGE and followed by Western blotting. The membrane was incubated with rabbit polyclonal anti-MET antibody and mouse polyclonal antiGAPDH as primary antibodies and IRDye $\AA$ 680CW Goat anti-Rabbit and IRDye ${ }^{\circledR} 680 \mathrm{CW}$ Goat anti-Mouse as secondary antibodies. GAPDH quantity was used as the loading control; (B) Densitometric values of the bands were calculated and normalized against background using GelAnalyzer 2010 software. Relative intensities (i.e. density ratio of target protein/ GAPDH) are represented in a bar diagram, with each bar depicting the mean \pm S.D. from 2 different analyses.

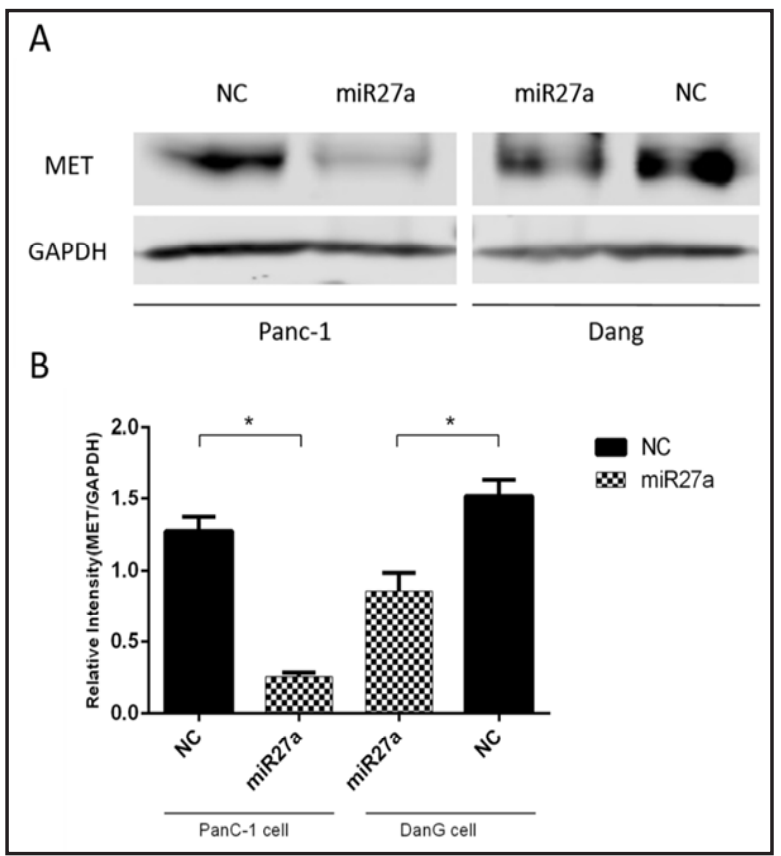

Next, we investigated the basal expression of RAR $\alpha, R A R \beta, R X R \alpha$ and RXR $\beta$ mRNAs in HPDE cells and PDAC cell lines. The results indicate that the expression of retinoid receptors varied in different cell lines: expression of RAR $\alpha$ was significantly decreased in BxPC-3, PanC1 and MiaPaCa-2 cells, but increased in DanG cells (Fig. 3). Similarly, RAR $\beta$ was significantly decreased in PanC-1, DanG and MiaPaCa-2 cells when compared to the level in the HPDE cells (Fig. 3). However, the expression of RAR $\beta$ was more than 8 times higher in BxPC-3 cells compared to HPDE cells (Fig. 3). In PanC-1 and MiaPaCa-2 cells, RXR $\alpha$ expressions were significantly down-regulated compared to HPDE cells. RXR $\beta$ expressions are decreased in all four PDAC cell lines compared to the HPDE cell line (Fig. 3).

No obvious correlation between selected miRNAs and retinoid receptor expression

Based on the basal expression of selected miRNAs and retinoid receptors in PDAC cell lines and HPDE cells, we next wanted to find out whether the expression of selected miRNAs correlated with the gene expression level of retinoid receptors. For this purpose, Spearman's rank correlation test was performed (Table 3). In HPDE cells and PDAC cell lines, expression levels of RAR $\beta$ were positively correlated with the level of miR-27a. Furthermore, expression levels of RXRA were also shown to be positively correlated with miR-206, miR-613 and miR138. However, no other significant correlations between the selected miRNAs and retinoid receptors were observed.

Despite the positive or absent correlations between retinoid receptors and miRNA expressions, we next wanted to investigate the effect of miR-27a, miR27b and miR9 levels on the gene expression of retinoid receptors due to the following facts: (1) the expression levels of miR-27a, miR27b, miR9, miR10a and miR10b in PDAC cell lines were significantly higher than that in HPDE cells; (2) The absolute expression levels of miR-27a, miR27b, miR9, miR10a and miR10b were not very low or even higher compared to U6 expression in PDAC cell lines and HPDE cells; (3) A real interaction between miR-27a and 3'UTP of RARA and RXRA has been demonstrated in rhabdomyosarcoma cells [33].

Transfection of PDAC cell lines with miR-27a, miR-27b and miR-9 does not influence retinoid receptor expression

To investigate the influence of miRNA on protein expression of the retinoid receptors, PDAC cells were transfected with miRNA mimics and incubated for 48 to $72 \mathrm{~h}$ under normal 
Fig. 6. Expression of retinoid receptors in Panc-1 and DanG cells transfected with antimiR27a, anti-miR27b or antimiR9. (A) $20 \mu \mathrm{g}$ total protein extracted from Panc-1 and DanG cell lines were electrophoresed in 10\% SDS-PAGE and followed by Western blotting. The membrane was incubated with rabbit polyclonal antibody (anti-RARa, antiRARb, anti-RXRa or anti-RXRb) as the primary antibody and IRDye $\AA$ 680CW Goat anti-Rabbit as the secondary antibody. GAPDH quantity was used as the loading control. (B) Expression of of various retinoid receptors depicted as relative intensities after the transfection of miRNA inhibitors. Densitometric values of the bands were calculated and normalized against background using GelAnalyzer 2010 software. Relative intensities (i.e. density ratio of target protein/GAPDH) are represented as bar diagrams, with each bar depicting the mean \pm S.D. from 2 different analyses.

Yin et al.: Mirna and Retinoid Receptors in PDAC

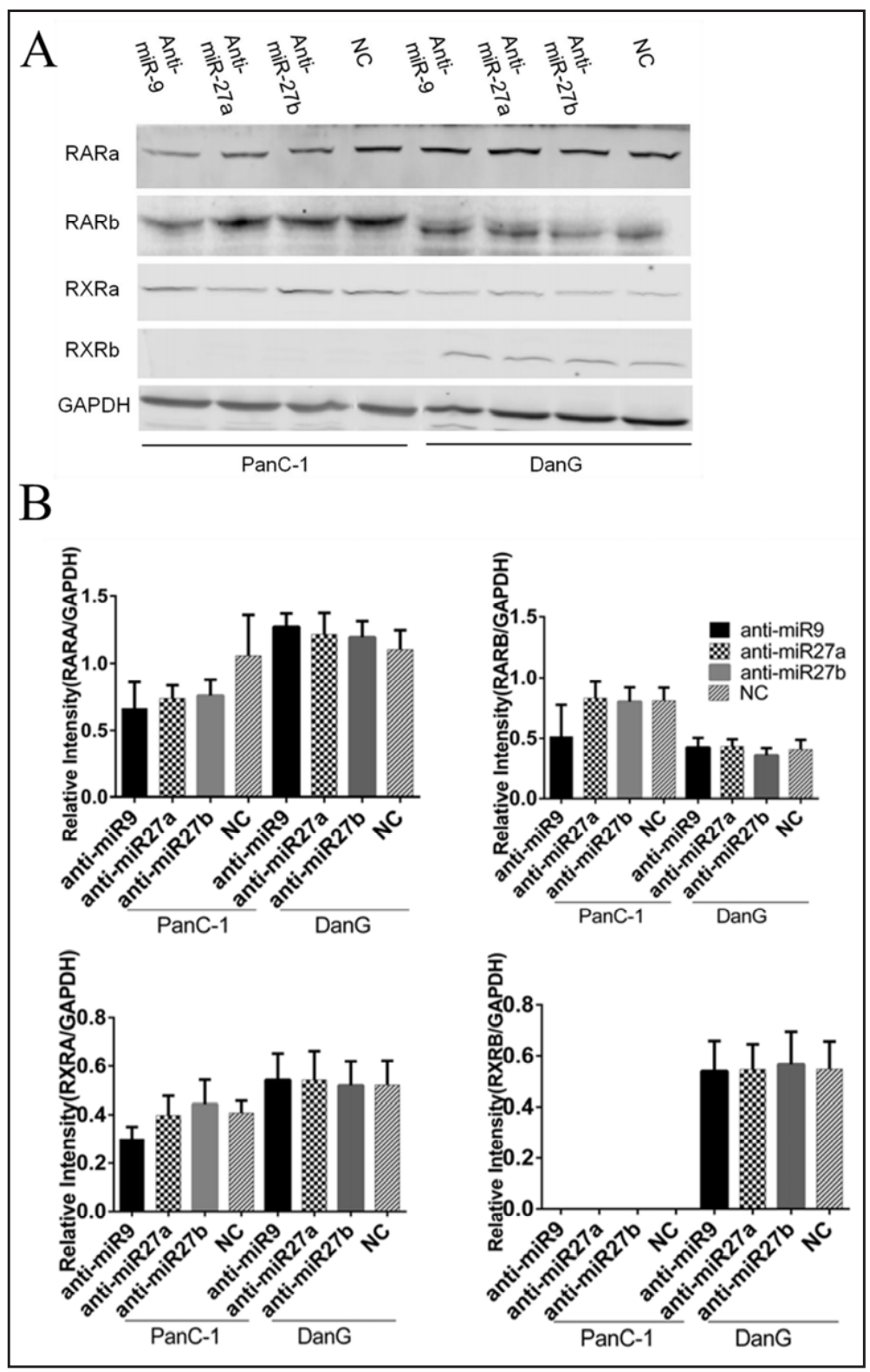

conditions for cell culture. Whole protein contents were isolated and Western blot analyses were carried out as described in the Material and Methods. Western blot analysis revealed that after transfection of miR-9, miR-27a or miR-27b, there were no significant inhibitions of retinoid receptor expressions in both PanC-1 and DanG cells compared with a negative control (Fig. 4).

To be sure that no technical problems have occurred, MET - an experimentally validated target of miR27a, was selected as a positive control [34]. The same amount of miR27a was transfected into PanC-1 and DanG cells with the same procedure, as mentioned above. After incubation for 48 to $72 \mathrm{~h}$ under normal conditions, whole protein contents were isolated and Western blot analyses were carried out as described in the Material and Methods. The analysis showed that transfection of miR27a mimics into PanC-1 and DanG cell lines led to a significant decrease in MET protein level (Fig. 5)

Transfection of miRNA inhibitors has no impact on retinoid receptor expressions

Finally, we investigated protein expression of retinoid receptors in PDAC cell lines after the transfection of miRNAs inhibitors. For this purpose, PDAC cells were transfected with 
miRNA inhibitor and incubated for 48 to $72 \mathrm{~h}$ under normal conditions for cell culture. Whole protein contents were isolated and Western blot analyses were carried out, as described in the Material and Methods. The analyses revealed no significant changes in the expression of retinoid receptors in both PanC- 1 cells and DanG cells compared with a negative control (Fig. 6).

\section{Discussion}

Consistent with our previous study, we showed in this report that retinoid receptors in PDAC cells are markedly down-regulated compared to normal pancreatic cells. The mechanisms by which retinoid receptors in cancer could be down-regulated include the following possibilities: (1) mutation or deletion of retinoid receptor genes; (2) transcriptional repression; (3) post-transcriptional repression. The fact that the retinoid receptor genes are not mutated in the majority of human cancer specimens indicates that epigenetic events could be involved. Studies have reported that RAR $\beta$ can be repressed by hypermethylation and histone deacetylation in some types of cancer, resulting in an associated resistance to the growth inhibitory effects of retinoic acid in some cancer types [35-37].

Regulation of retinoid receptor genes on post-transcriptional level can also contribute to the dysregulated retinoid receptor expression in PDAC. Among these mechanisms, miRNA serves as a major pattern regulator of up to $30 \%$ of the protein-coding genes in mammals. Regulation by miRNAs result in the destabilization of target mRNA, translational repression and even activation [38].

In this study, we aimed to identify up-regulated miRNAs, which could target retinoid receptors in PDAC cell lines. We found that the selected up-regulated miRNAs (miR27a, miR27b and miR9) were not associated with the down-regulation of retinoid receptors. There were significant correlations between specific miRNA and retinoid receptors, such as the correlation between miR-613 and RXRA, but the correlation coefficient reveals that they are positively correlated rather than negatively correlated. As such, we cannot draw the conclusion that there are significant correlations that are meaningful for our experiments. Since a single miRNA has a lot of putative targets in a particular cell, we can assume that this pleotropic effect of miRNAs manifested in the modulation of expression of some proteins involved in the regulation of retinoid receptor expression.

However, the high expression of miR27, miR-21and miR-23a in PDAC patients was associated with aggressive tumor behavior and poor survival after PDAC resection. Specifically, miR-27a was found to target BTG2, ZBTB10 and Spry2, which are associated with cancer survival, growth and angiogenic responses in PDAC [39]. It was concluded that the triple combination of miR-21/23a/27a could be a prognosis factor of PDAC. These results showing that miR-27a is significantly up-regulated in PDAC cell lines are inconsistent with our results. Interestingly, Tombolan and his colleagues demonstrated that miR-27a is implicated in tumorigenesis as it targets RAR $\alpha$ and RXR $\alpha$ expression in the 293T cell line [33]. A reason for this finding could be that specific miRNAs can have different effects in particular cell types and tumor entities.

Based on these findings, we conclude that miR-27a, miR-27b, miR-9, miR10a and miR$10 \mathrm{~b}$ are up-regulated in PDAC cells compared to HPDE cells. The up-regulation of these miRNAs is not responsible for the down-regulation of $R A R \alpha, R A R \beta, R X R \alpha$ and $R X R \beta$ in PDAC cells.

\section{Disclosure Statement}

The authors declare that there are no conflicts of interest. 


\section{Cellular Physiology Cell Physiol Biochem 2017;44:644-656 \begin{tabular}{ll|l} 
DOI: 10.1159/000485276 & $\begin{array}{l}\text { O 2017 The Author(s). Published by S. Karger AG, Basel } \\
\text { www.karger.com/cpb }\end{array}$ \\
\hline and Biochemistry Published online: November 23, 2017
\end{tabular}}

Yin et al.: Mirna and Retinoid Receptors in PDAC

\section{Acknowledgements}

We thank Ms. Sevdije Gashi for her excellent technical assistance and Dr. Serene Lee for proofreading the manuscript. We would like to thank our anonymous reviewers for their excellent suggestions and comments that helped to improve our work.

\section{References}

1 Cipolla GA: A non-canonical landscape of the microRNA system. Front Genet 2014;5:337.

-2 van Kouwenhove M, Kedde M, Agami R: MicroRNA regulation by RNA-binding proteins and its implications for cancer. Nat Rev Cancer 2011;11:644-656.

-3 Rana TM: Illuminating the silence: understanding the structure and function of small RNAs. Nat Rev Mol Cell Biol 2007;8:23-36.

4 Stefani G, Slack FJ: Small non-coding RNAs in animal development. Nat Rev Mol Cell Biol 2008;9:219-230.

5 Truesdell SS, Mortensen RD, Seo M, Schroeder JC, Lee JH, LeTonqueze O, Vasudevan S: MicroRNA-mediated mRNA translation activation in quiescent cells and oocytes involves recruitment of a nuclear microRNP. Sci Rep 2012;2:842.

6 Lytle JR, Yario TA, Steitz JA: Target mRNAs are repressed as efficiently by microRNA-binding sites in the 5' UTR as in the 3' UTR. Proc Natl Acad Sci U S A 2007;104:9667-9672.

-7 Lee I, Ajay SS, Yook JI, Kim HS, Hong SH, Kim NH, Dhanasekaran SM, Chinnaiyan AM, Athey BD: New class of microRNA targets containing simultaneous 5'-UTR and 3'-UTR interaction sites. Genome Res 2009;19:1175-1183.

-8 Henke JI, Goergen D, Zheng J, Song Y, Schuttler CG, Fehr C, Junemann C, Niepmann M: microRNA-122 stimulates translation of hepatitis C virus RNA. EMBO J 2008;27:3300-3310.

-9 Orom UA, Nielsen FC, Lund AH: MicroRNA-10a binds the 5'UTR of ribosomal protein mRNAs and enhances their translation. Mol Cell 2008;30:460-471.

-10 Tsai NP, Lin YL, Wei LN: MicroRNA mir-346 targets the 5'-untranslated region of receptor-interacting protein 140 (RIP140) mRNA and up-regulates its protein expression. Biochem J 2009;424:411-418.

11 Moretti F, Thermann R, Hentze MW: Mechanism of translational regulation by miR-2 from sites in the 5' untranslated region or the open reading frame. RNA 2010;16:2493-2502.

12 Jin Y, Wang C, Liu X, Mu W, Chen Z, Yu D, Wang A, Dai Y, Zhou X: Molecular characterization of the microRNA-138-Fos-like antigen 1 (FOSL1) regulatory module in squamous cell carcinoma. J Biol Chem 2011;286:40104-40109.

13 Amann PM ES, Schmidt J, Bazhin AB: Regulation of gene expression by retinoids. Curr Med Chem 2011;18:1405-1412.

14 Hartwig W, Werner J, Jager D, Debus J, Buchler MW: Improvement of surgical results for pancreatic cancer. Lancet Oncol 2013;14:e476-485.

15 Rosty C, Goggins M: Early detection of pancreatic carcinoma. Hematol Oncol Clin North Am 2002;16:37-52.

16 Bazhin AV, Shevchenko I, Umansky V, Werner J, Karakhanova S: Two immune faces of pancreatic adenocarcinoma: possible implication for immunotherapy. Cancer Immunol Immunother 2014;63:59-65.

-17 Sun XJ, Liu BY, Yan S, Jiang TH, Cheng HQ, Jiang HS, Cao Y, Mao AW: MicroRNA-29a Promotes Pancreatic Cancer Growth by Inhibiting Tristetraprolin. Cell Physiol Biochem 2015;37:707-718.

18 Yang W, Yang Y, Xia L, Yang Y, Wang F, Song M, Chen X, Liu J, Song Y, Zhao Y, Yang C: MiR-221 Promotes Capan-2 Pancreatic Ductal Adenocarcinoma Cells Proliferation by Targeting PTEN-Akt. Cell Physiol Biochem 2016;38:2366-2374.

19 Song W, Li Q Wang L, Wang L: Modulation of Fox01 expression by miR-21 to promote growth of pancreatic ductal adenocarcinoma. Cell Physiol Biochem 2015;35:184-190.

20 Song B, Zhang C, Li G, Jin G, Liu C: MiR-940 inhibited pancreatic ductal adenocarcinoma growth by targeting MyD88. Cell Physiol Biochem 2015;35:1167-1177.

21 Song B, Zheng K, Ma H, Liu A, Jing W, Shao C, Li G, Jin G: miR-429 determines poor outcome and inhibits pancreatic ductal adenocarcinoma growth by targeting TBK1. Cell Physiol Biochem 2015;35:1846-1856. 


\section{Cellular Physiology Cell Physiol Biochem 2017;44:644-656 \begin{tabular}{l|l|l} 
DOI: 10.1159/000485276 & O 2017 The Author(s). Published by S. Karger AG, Basel \\
www.karger.com/cpb
\end{tabular} \\ Yin et al.: Mirna and Retinoid Receptors in PDAC}

22 Zhang Y, Guan DX, Shi J, Gao H, Li JJ, Zhao JS, Qiu L, Liu J, Li N, Guo WX, Xue J, Zhou FG, Wu MC, Wang HY, Xie D, Cheng SQ: All-trans retinoic acid potentiates the chemotherapeutic effect of cisplatin by inducing differentiation of tumor initiating cells in liver cancer. J Hepatol 2013;59:1255-1263.

23 Guan J, Zhang H, Wen Z, Gu Y, Cheng Y, Sun Y, Zhang T, Jia C, Lu Z, Chen J: Retinoic acid inhibits pancreatic cancer cell migration and EMT through the downregulation of IL-6 in cancer associated fibroblast cells. Cancer Lett 2014;345:132-139.

-24 Bleul T, Ruhl R, Bulashevska S, Karakhanova S, Werner J, Bazhin AV: Reduced retinoids and retinoid receptors' expression in pancreatic cancer: A link to patient survival. Mol Carcinog 2015;54:870-879.

25 Bazhin AV, Bleul T, de Lera AR, Werner J, Ruhl R: Relationship Between All-trans-13, 14-Dihydro Retinoic Acid and Pancreatic Adenocarcinoma. Pancreas 2016;45:e29-31.

-26 Bazhin AV, Tambor V, Dikov B, Philippov PP, Schadendorf D, Eichmuller SB: cGMP-phosphodiesterase 6, transducin and Wnt5a/Frizzled-2-signaling control cGMP and $\mathrm{Ca}(2+)$ homeostasis in melanoma cells. Cell Mol Life Sci 2010;67:817-828.

27 Schmittgen TD, Livak KJ: Analyzing real-time PCR data by the comparative C(T) method. Nat Protoc 2008;3:1101-1108.

28 Wang X, El Naqa IM: Prediction of both conserved and nonconserved microRNA targets in animals. Bioinformatics 2008;24:325-332.

29 Wang X: Improving microRNA target prediction by modeling with unambiguously identified microRNAtarget pairs from CLIP-ligation studies. Bioinformatics 2016;32:1316-1322.

-30 Wong N, Wang X: miRDB: an online resource for microRNA target prediction and functional annotations. Nucleic Acids Res 2015;43:D146-152.

-31 Agarwal V, Bell GW, Nam JW, Bartel DP: Predicting effective microRNA target sites in mammalian mRNAs. Elife 2015;4.

-32 Krek A, Grun D, Poy MN, Wolf R, Rosenberg L, Epstein EJ, MacMenamin P, da Piedade I, Gunsalus KC, Stoffel M, Rajewsky N: Combinatorial microRNA target predictions. Nat Genet 2005;37:495-500.

-33 Tombolan L, Zampini M, Casara S, Boldrin E, Zin A, Bisogno G, Rosolen A, De Pitta C, Lanfranchi G: MicroRNA-27a Contributes to Rhabdomyosarcoma Cell Proliferation by Suppressing RARA and RXRA. PLoS One 2015;10:e0125171.

34 Acunzo M, Romano G, Palmieri D, Lagana A, Garofalo M, Balatti V, Drusco A, Chiariello M, Nana-Sinkam P, Croce CM: Cross-talk between MET and EGFR in non-small cell lung cancer involves miR-27a and Sprouty2 Proc Natl Acad Sci U S A 2013;110:8573-8578.

-35 Sirchia SM, Ferguson AT, Sironi E, Subramanyan S, Orlandi R, Sukumar S, Sacchi N: Evidence of epigenetic changes affecting the chromatin state of the retinoic acid receptor beta2 promoter in breast cancer cells. Oncogene 2000;19:1556-1563.

-36 Kim YS, Kim E, Park YJ, Kim Y: Retinoic acid receptor beta enhanced the anti-cancer stem cells effect of beta-carotene by down-regulating expression of delta-like 1 homologue in human neuroblastoma cells. Biochem Biophys Res Commun 2016;480:254-260.

-37 Lee MF, Hsieh NT, Huang CY, Li CI: All Trans-Retinoic Acid Mediates MED28/HMG Box-Containing Protein 1 (HBP1)/beta-Catenin Signaling in Human Colorectal Cancer Cells. J Cell Physiol 2016;231:1796-1803.

-38 Huntzinger E, Izaurralde E: Gene silencing by microRNAs: contributions of translational repression and mRNA decay. Nat Rev Genet 2011;12:99-110.

-39 Frampton AE, Castellano L, Colombo T, Giovannetti E, Krell J, Jacob J, Pellegrino L, Roca-Alonso L, Funel N, Gall TM, De Giorgio A, Pinho FG, Fulci V, Britton DJ, Ahmad R, Habib NA, Coombes RC, Harding V, Knosel T, Stebbing J, Jiao LR: MicroRNAs cooperatively inhibit a network of tumor suppressor genes to promote pancreatic tumor growth and progression. Gastroenterology 2014;146:268-277 e218. 